\title{
The application of Pressure-regulated volume controlled ventilation mode in Video-assisted Thoracoscopic Surgery pulmonary segmentectomy
}

qian han ( $\square$ tongxunzuozhe1984@163.com )

Cangzhou People's Hospital

Wenyu Yao

cangzhou central hospital

Chuansuo Zhang

Cangzhou central College

Shuishui Wu

cangzhou central hospital

Haijun Chen

cangzhou central hospital

Research article

Keywords: Pressure-regulated volume controlled ventilation mode, One lung ventilation, Volumecontrolled ventilation mode, Pulmonary segmentectomy

Posted Date: December 23rd, 2019

DOl: https://doi.org/10.21203/rs.2.19447/v1

License: (c) (1) This work is licensed under a Creative Commons Attribution 4.0 International License.

Read Full License 


\section{Abstract}

Background: Pressure-regulated capacity control volume controlled (PRVC) ventilation mode is a new type of ventilation mode, which could reduce the occurrence of ventilator-related lung injury. The aim of this study was to explore PRVC mode on airway pressure, oxygenation index, pulmonary inflammatory index and prognosis in patients with one-lung ventilation during pulmonary segmentectomy.

Methods: Eighty ASA $\mathbb{\bigotimes}-\mathbb{Q}$ Patients with moderate to severe pulmonary dysfunction, ready to receive segmentectomy were randomly divided into VC and PRVC group, which included 40 patients in each group. PRVC ventilation mode was performed for patients in VC-group in the first 5 minutes after OLV, and then ventilation mode was transformed into VC ventilation mode till the end of operation. In PRVC-group, ventilation modes were performed in opposite order. The changes of airway peak pressure, airway platform pressure, pulmonary static compliance, blood gas analysis results and hemodynamics in the two groups under different ventilation modes were recorded. The whole blood samples and bronchoalveolar lavage fluid (BALF) in ventilated lung were collected to determine the level of TNF-a, IL$1 \beta$, IL-6 and IL-8 after the surgery.

Results: Both static lung compliance and the peak expiratory pressure in PRVC-group were significantly lower than those in $\mathrm{VC}$-group $(\mathrm{P}=0.023)$. But there were no difference in hemodynamic parameters such as heart rate, blood pressure. The arterial blood gas analysis ( $\mathrm{pH}, \mathrm{pO} 2$ and $\mathrm{pCO} 2)$ between the two groups during, as well as postoperative pulmonary complications and length of hospital stay also did not show difference. However, the levels of TNF-a, IL-6, IL-8 and IL-10 in BALF in VC-group were significantly higher than that in PRVC-group $(P=0.01)$.

Conclusion: PRVC ventilation mode during intraoperative one-lung ventilation can effectively relieve airway pressure and reduce the secretion of inflammatory factors in the lung, which is a safe and protective ventilation mode for patients with poor preoperative pulmonary function who undergo pulmonary segmentectomy.

\section{Background}

With the implementation of protective ventilation strategy in one-lung ventilation, the incidence of intraoperative ventilator-associated lung injury has significantly reduced less than before [1-4]. However, due to low of preoperative presence of pulmonary compliance and high airway reactivity, many patients still exist high airway pressure with Volume -controlled (VC) ventilation mode in the one-lung ventilation, which will increase postoperative pulmonary complications. Nowadays, Pressure-regulated capacity control volume controlled (PRVC) ventilation mode is a new clinical emerging in recent years, which combines pressure control ventilation and capacity control together [5]. The advantage of PRVC can readjust pressure and volume control according to the patient's lung compliance, which ensures preset tidal volume delivery. It can make the distribution of gas in the lung evenly, and get better alveolar ventilation, which could reduce airway pressure while maintaining ventilation. Many research reported 
that PRVC mode can reduce lung injury of patients due to mechanical ventilation is slower in terms of lung compliance, especially to chronic obstructive pulmonary disease [6]. In recent ten years, with the widespread of the lung cancer CT-screening in imaging modalities, a large number of ground-glass opacity (GGO) and small solid lung tumor $(\leq 2 \mathrm{~cm}$ ) had bee diagnosed and sublobar resection, especially segmentectomy, was used more extensively than before.

Our study aims to PRVC ventilation mode on airway pressure during the operation of pulmonary segmentectomy with poor pulmonary function and oxygenation index, lung inflammation response as well as clinical prognostic factors were evaluated by compared with conventional VC Model .

\section{Patients And Methods}

\section{Patients}

This retrospective study comprised of 80 patients with ASA $\mathbb{X}-\mathbb{Z}$, moderate to severe pulmonary dysfunction who underwent pulmonary segmentectomy in VATS at our hospital between January 2017 and January 2018 (Table 1). In the present study, moderate to severe pulmonary dysfunction means that preoperative forced vital capacity $(\mathrm{FVC})$ and forced expiratory volume in one second $\left(\mathrm{FEV}_{1}\right)$ were required to be under $60 \%$ of the predicted value, and the $\mathrm{FEV}_{1} / \mathrm{FVC}$ ratio was required to be low than $50 \%$. Patients who used to undergo the surgery of lung or heart or had the medical history of chest deformity or tuberculosis in the past were excluded from the present study. These patients were divided into two groups based on the reconstruction method: PRVC-group and VC-group. The present study was approved by the Research Ethics Committee of HeBei Medical University (People's Republic of China; Approval number: HDLL201776865), and written informed consent was obtained from all patients. 
Table 1

Patient demographics and clinical data

\begin{tabular}{|c|c|c|}
\hline Variable & Group -VC & Group -PRVC \\
\hline Patients & 40 & 40 \\
\hline \multicolumn{3}{|l|}{ Age (years) } \\
\hline$\leq 60$ & 23 & 22 \\
\hline$>60$ & 17 & 18 \\
\hline \multicolumn{3}{|l|}{ Gender } \\
\hline male & 22 & 19 \\
\hline Female & 18 & 21 \\
\hline \multicolumn{3}{|l|}{ ASA level } \\
\hline 1 & 21 & 18 \\
\hline II & 19 & 22 \\
\hline \multicolumn{3}{|l|}{ Segmentectomy Side } \\
\hline Right & 22 & 19 \\
\hline left & 18 & 21 \\
\hline Poor cardiac function ${ }^{2}$ & 17 & 14 \\
\hline COPD & 6 & 8 \\
\hline FVC\% & $52 \pm 6$ & $51 \pm 5$ \\
\hline $\mathrm{FEV}_{1} \%$ & $48 \pm 3$ & $47 \pm 3$ \\
\hline $\mathrm{FEV}_{1} / \mathrm{FVC} \%$ & $43 \pm 2$ & $41 \pm 2$ \\
\hline Surgery time(min) & $107 \pm 12$ & $113 \pm 13$ \\
\hline OLV time & $93 \pm 10$ & $88 \pm 9$ \\
\hline
\end{tabular}

\section{Anesthesia procedure}

All the patients were performed by electrocardiogram before operation to record basic Heart rate, blood pressure, pulse and $\mathrm{SpO}_{2}$. Epidural puncture and catheterization were performed between thoracic vertebrae 7-8, with $3 \mathrm{~mL} 1 \%$ lidocaine injected as the experimental dose. Then, we performed internal jugular vein, radial artery puncture catheterization which could collect Arterial blood for blood gas 
analysis. The patient was routinely anesthetized by double - chamber endotracheal intubation using a Maquet Flow-3.0 (Maquet Medical Equipment, Shanghai, China) ventilator for mechanical ventilation.

\section{Methods}

After one-lung ventilation, patients in the VC-group underwent PRVC mode for $5 \mathrm{~min}$, and airway peak pressure and pulmonary static compliance were recorded. After the arterial blood gas analysis was detected, the ventilation mode was changed to VC mode until the single lung ventilation node in the end. After 25 min of ventilation in VC mode, airway peak pressure, platform pressure and pulmonary static compliance were recorded, and arterial blood gas was analyzed. In the PRVC-group, one-lung ventilation was performed for $5 \mathrm{~min}$ in the $\mathrm{VC}$ mode, and airway peak pressure, platform pressure and pulmonary static compliance were recorded. Arterial blood gas analysis was performed after $5 \mathrm{~min}$, and the ventilation mode was switched to PRVC mode under the condition that the ventilation parameters remained unchanged until the end of single-lung ventilation.

Bronchoalveolar lavage fluid (BALF) was collected for analysis of inflammatory factors in all patients before the end of one-lung ventilation. The lavage solution was rapidly filtered and removed with a double layer of sterile gauze, and the supernatant was removed after centrifugation. TNF-a, IL-6, IL-8 and IL-10 were detected according to the instructions of ELISA kit. Meanwhile, one $\mathrm{mL}$ peripheral blood also was taken to detect TNF-a, IL-6, IL-8 and IL-10. Hospital stay, ICU stay and postoperative pulmonary complications were recorded to compare the difference between groups.

\section{Statistical analysis}

All statistical analyses were performed using SPSS 20.0 software (IBM, Armonk, NY, USA). Anova analysis was used for comparison of hemodynamic indicators. Single-factor anova was used for comparison of blood gas analysis results and inflammatory index. Chi-square test was applied to comparison of counting data. A two-sided $p$ value $<0.05$ was considered to be statistically significant.

\section{Results}

\section{clinicopathologic data}

As showed in Table 1, there was no significant difference between the two groups in age, gender, body mass index (BMI), ASA classification, surgical location, pulmonary disease history, preoperative pulmonary function index, operative time, single lung ventilation time, intraoperative blood loss and intraoperative infusion volume $(P>0.05)$.

Ventilation patterns on airway pressure, lung compliance, and oxygenation index between two groups

Patients with PRVC mode ventilation had significantly lower mean airway peak pressure and higher pulmonary static compliance, but the difference in oxygenation index between groups was not statistically significant (Table 2). 
Table 2

ventilation patterns on airway pressure, lung compliance, and oxygenation index in two groups

\begin{tabular}{|c|c|c|c|c|c|c|}
\hline & \multicolumn{3}{|c|}{ PRVC group } & \multicolumn{3}{|l|}{ VC group } \\
\hline & VC mode & $\begin{array}{l}\text { PRVC } \\
\text { mode }\end{array}$ & $\begin{array}{l}P \\
\text { value }\end{array}$ & $\begin{array}{l}\text { PRVC } \\
\text { mode }\end{array}$ & VC mode & $\begin{array}{l}P \\
\text { value }\end{array}$ \\
\hline $\begin{array}{l}\text { Plateau } \\
\text { Expiratory } \\
\text { pressure } \\
\left(\mathrm{p} / \mathrm{cm} \mathrm{H}_{2} \mathrm{O}\right)\end{array}$ & $15.6 \pm 2.3$ & & & & $17.9 \pm 2.6$ & \\
\hline $\begin{array}{l}\text { Peak } \\
\text { expiratory } \\
\text { pressure } \\
\left(\mathrm{p} / \mathrm{cm} \mathrm{H}_{2} \mathrm{O}\right)\end{array}$ & $26.5 \pm 2.6$ & $18.1 \pm 2.3$ & 0.03 & $17.9 \pm 2.3$ & $24.6 \pm 2.5$ & 0.025 \\
\hline $\begin{array}{l}\text { Static } \\
\text { lung compliance/L·cm } \\
\mathrm{H}_{2} \mathrm{O}^{-1}\end{array}$ & $24.6 \pm 2.6$ & $31.5 \pm 2.8$ & 0.034 & $30.6 \pm 2.7$ & $24.5 \pm 2.4$ & 0.02 \\
\hline $\mathrm{pH}$ & $\begin{array}{l}7.39 \pm \\
0.04\end{array}$ & $\begin{array}{l}7.38 \pm \\
0.02\end{array}$ & $>0.05$ & $\begin{array}{l}7.35 \pm \\
0.02\end{array}$ & $\begin{array}{l}7.38 \pm \\
0.04\end{array}$ & $>0.05$ \\
\hline $\mathrm{O} 2$ pressure $\mathrm{p} / \mathrm{mm} \mathrm{Hg}$ & $39 \pm 3$ & $39 \pm 5$ & $>0.05$ & $37 \pm 2$ & $39 \pm 3$ & $>0.05$ \\
\hline $\mathrm{CO} 2$ pressure $\mathrm{p} / \mathrm{mm} \mathrm{Hg}$ & $160 \pm 53$ & $161 \pm 60$ & $>0.05$ & $164 \pm 55$ & $161 \pm 55$ & $>0.05$ \\
\hline $\begin{array}{l}\text { Oxygenation index } \mathrm{p} / \mathrm{mm} \\
\mathrm{Hg}\end{array}$ & $223 \pm 97$ & $238 \pm 56$ & $>0.05$ & $228 \pm 87$ & $233 \pm 92$ & $>0.05$ \\
\hline
\end{tabular}

\section{Ventilation patterns on inflammatory marks of lung and plasma}

To understand whether different ventilation patterns affect inflammatory responses in the lungs and throughout the body, TNF-a, IL-6, IL-8 and IL-10 levels in the lung and peripheral blood of the aerated side were measured. The results showed that the levels of TNF-a, IL-6, IL-8 and IL-10 in the BALF and plasma in the PRVC group were significantly lower than those in the VC group (Fig. 1).

\section{Ventilation pattern on postoperative pulmonary complications and length of hospital stay}

To understand the effect of different ventilation patterns on the prognosis of patients, we collected the incidence of postoperative pulmonary complications and the length of hospital stay. In our study, there was no difference in the incidence of perioperative pulmonary complications between the two groups. The average length of hospital stay in VC-group was longer than PRVC-group $(p=0.03)$. 


\section{Discussion}

Nowadays, VC mode is mostly used for ventilation in surgical operation. However, in the case of singlelung ventilation in VATS pulmonary segmentectomy, some patients may suffer from excessive airway pressure due to poor lung compliance and high airway resistance on the ventilated side. Therefore, protective pulmonary ventilation strategy should be adopted, that is, neap volume $(4-6 \mathrm{~mL} / \mathrm{kg})$ combined with low level PEEP (usually $5 \mathrm{~cm} \mathrm{H}_{2} \mathrm{O}$ ) [2,3]. This strategy avoids excessive airway pressure to a certain extent, but there are still a few patients suffered high airway pressure under the condition of good

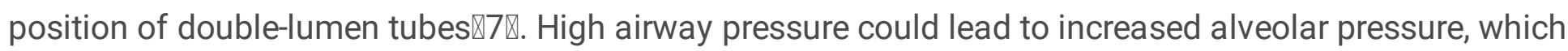
can reduce the amount of blood returned to the heart and then cause a drop in blood pressure [4, 7]. Meanwhile, it can also induce airway injury, leading to acute lung injury and postoperative pulmonary complications.

In the traditional VC mode, the ventilator relies on preset tidal volume, inspiratory time and breathing frequency to ensure the patient's ventilation volume, and the size of the airway pressure is mainly affected by the patient's airway resistance and lung compliance [8]. In order to achieve the preset tidal volume, the alveolar ventilation of lung tissues with different compliance is uneven, which is likely to cause structural damage of lung parenchyma and pulmonary mesenchyme, leading to airway injury [9]. According to the lung protective ventilation strategy, the goal of ventilation management is to minimize ventilator-associated lung injury, and PRVC mode is well consistent with this strategy $[10,11]$. On the basis of ensure the preset tidal volume and respiratory function of automatic continuous monitoring lung compliance and volume/pressure relationship, PRVC ventilation mode reduce airway pressure as much as possible, so as to reduce the pressure of the positive pressure ventilation [12]. PRVC ventilation for the first time for experimental ventilation, inspiratory pressure is low $\left(5 \mathrm{~cm} \mathrm{H} \mathrm{H}_{2} \mathrm{O}\right)$. The lung compliance and inspiratory pressure to reach the preset tidal volume was calculated by the microcomputer measure. The actual inspiration pressure of the next ventilation is $75 \%$ of the above calculated value. After several ventilation, the actual tidal volume can be consistent with the preset tidal volume [13, 14]. Since the waveform of inspiratory velocity is a deceleration wave, the vortex of gas can be reduced when the patient's airway resistance is large, so the pressure consumption and peak inspiratory pressure can be reduced while the preset tidal volume is ensured [15]. It has been reported that PRVC mode can better meet the requirement of tidal volume during mechanical ventilation and reduce the airway pressure as well as lung complications $₫ 16 \bigotimes$.

In this study, the changes of respiratory mechanics, oxygenation index and respiratory mechanics under different ventilation modes in one-lung-ventilation were compared by using cross design method. The results indicated that, when using PRVC mode for ventilation, not only the airway pressure level was significantly lower than that of VC mode, but also the pulmonary static compliance was significantly higher than that of $\mathrm{VC}$ mode. In addition, the detection of inflammatory indicators in the lung showed that the levels of TNF-, IL-6, IL-8 and IL-10 from BALF in the PRVC group were significantly lower than those in the VC group, suggesting that excessive airway pressure may lead to the structural destruction of lung 
tissue with poor compliance, thus causing airway injury. This result is consistent with the results of related literatures [17-20].

There was no significant difference in heart rate, blood pressure, oxygenation index and incidence of postoperative pulmonary complications between the two groups $(P>0.05)$ (Table 3$)$. Further analysis shows, 9 cases of postoperative non-infectious acute lung injury (both in VC-group) with intraoperative airway pressure for a long time was up to $30 \mathrm{~cm} \mathrm{H}_{2} \mathrm{O}$, which may be important reasons of these patients appear postoperative respiratory dysfunction. Therefore, the use of intraoperative protective ventilation strategy to reduce the airway pressure is of great significance.

Table 3

pulmonary complications and length of hospital stay between groups

\begin{tabular}{|llll|}
\hline Variable & Group -VC & Group -PRVC & P value \\
\hline Respiratory dysfunction & 2 & 1 & $>0.05$ \\
\hline pulmonary infection & 3 & 2 & $>0.05$ \\
\hline Pulmonary atelectasis & 4 & 2 & $>0.05$ \\
\hline Acute lung injury & 6 & 2 & $>0.05$ \\
\hline Re-intubation & 1 & 0 & $>0.05$ \\
\hline ICU stay t/d $(\mathrm{x} \pm \mathrm{s})$ & $1.9 \pm 0.7$ & $1.3 \pm 0.5$ & 0.045 \\
\hline Hospital stay $\mathrm{t} / \mathrm{d}(\mathrm{x} \pm \mathrm{s})$ & $7.6 \pm 1.5$ & $5.4 \pm 1.2$ & 0.03 \\
\hline
\end{tabular}

In a conclusion, PRVC model has the advantages of lowering airway pressure and improving lung compliance, which could reduce the single lung ventilation during acute lung injury caused by high pressure. Therefore, PRVC mode is a good choice for patients with poor pulmonary ventilation function in VATS pulmonary segmentectomy (such as chronic bronchitis, COPD, etc.) during the operation, especially for single-lung ventilation.

\section{Abbreviations}

VATS:Video-assisted Thoracoscopic Surgery; PRVC:Pressure-regulated volume controlled; VC:Volume controlled; OLV:One-lung ventilation; BALF:Bronchoalveolar lavage fluid; FVC:Forced vital capacity; FEV1:Forced expiratory volume in one second

\section{Declarations}

\section{Acknowledgements}


None.

\section{Authors' contributions}

QH conceived the study, analyzed the collected data and drafted the manuscript. WY and CS participated in the protocol conception and corrected the manuscript. QH , SW and $\mathrm{CH}$ collected and analyzed the data, and drafted the manuscript. All authors have read and approved the final manuscript before publication.

\section{Funding}

None.

\section{Availability of data and materials}

Because of the secrecy policy restrictions on military university, the datasets used in this study are not allowed to be deposited in publicly. The datasets are available from the corresponding author on reasonable request.

\section{Ethics approval and consent to participate}

The present study was approved by the Research Ethics Committee of HeBei Medical University (People's Republic of China; Approval number: HDLL201776865), and written informed consent was obtained from all patients.

\section{Consent for publication}

Not applicable.

\section{Competing interests}

All authors declare that they have no competing interest in relation with the subject of the manuscript.

\section{References}

1. Kao RL, Huang W, Martin CM, et al. The effect of aerosolized indomethacin on lung inflammation and injury in a rat model of blunt chest trauma. Can J Surg. 2018 Dec 1;61(6):S208-S218.

2. Willson DF, Hall M, Beardsley A, et al. Pediatric Ventilator-Associated Events: Analysis of the Pediatric Ventilator-Associated Infection Data. Pediatr Crit Care Med. 2018 Dec;19(12):e631-e636.

3. Serpa Neto A, Cardoso SO, Manetta JA, et al. Association between use of lung-protective ventilation with lower tidal volumes and clinical outcomes among patients without acute respiratory distress syndrome: a meta-analysis. JAMA, 2012, 308(16): 1651-1659. 
4. Dhar SM, Breite MD, Barnes SL, et al. Pulmonary Contusion in Mechanically Ventilated Subjects After Severe Trauma. Respir Care. 2018 Aug;63(8):950-954.

5. Aghadavoudi O, Alikiaii B, Sadeghi F. Comparison of respiratory and hemodynamic stability in patients with traumatic brain injury ventilated by two ventilator modes: Pressure regulated volume control versus synchronized intermittent mechanical ventilation. Adv Biomed Res. $2016 \mathrm{Nov}$ 28;5:175.

6. Hausman MS Jr, Jewell ES, Engoren M. Regional versus general anesthesia in surgical patients with chronic obstructive pulmonary disease: does avoiding general anesthesia reduce the risk of postoperative complications? Anesth Analg. 2015 Jun;120(6):1405-12.

7. Spaeth J, Ott M, Karzai W, et al. Double-lumen tubes and auto-PEEP during one-lung ventilation. $\mathrm{Br} \mathrm{J}$ Anaesth. 2016 Jan;116(1):122-30.

8. Buehler S, Schumann S, Vimláti L, et al. Simultaneous monitoring of intratidal compliance and resistance in mechanically ventilated piglets: A feasibility study in two different study groups. Respir Physiol Neurobiol. 2015 Dec;219:36-42.

9. McCoy AM, Herington JL, Stouch ANIKK, et al. $\beta$ Activation in the Fetal Lung Mesenchyme Alters Lung Vascular Development but Not Airway Morphogenesis. Am J Pathol. 2017 Dec;187(12):26352644.

10. Reiterer F, Schwaberger B, Freidl T, et al. Lung-protective ventilatory strategies in intubated preterm neonates with RDS.,Paediatr Respir Rev. 2017 Jun;23:89-96.

11. Costa Leme A, Hajjar LA, Volpe MS, et al. Effect of Intensive vs Moderate Alveolar Recruitment Strategies Added to Lung-Protective Ventilation on Postoperative Pulmonary Complications: A Randomized Clinical Trial.JAMA. 2017 Apr 11;317(14):1422-1432.

12. Samantaray A, Hemanth N. Comparison of two ventilation modes in post-cardiac surgical patients.Saudi J Anaesth. 2011 Apr;5(2):173-8.

13. Lee KB, Kim MK, Jeong JR, et al. Reliability of an Electronic Inspiratory Loading Device for Assessing Pulmonary Function in Post-Stroke Patients. Med Sci Monit. 2016 Jan 19;22:191-6.

14. Samantaray A, Hemanth N. Comparison of two ventilation modes in post-cardiac surgical patients. Saudi J Anaesth, 2011, 5(2): 173-178.

15. Mahmoud K, Ammar A, Kasemy Z. Comparison Between Pressure-Regulated Volume-Controlled and Volume-Controlled Ventilation on Oxygenation Parameters, Airway Pressures, and Immune Modulation During Thoracic Surgery. J Cardiothorac Vasc Anesth. 2017 Oct;31(5):1760-1766.

16. Montes FR, Pardo DF, Charrís $\mathrm{H}$, et al. Comparison of two protective lung ventilatory regimes on oxygenation during one-lung ventilation: a randomized controlled trial. J Cardiothorac Surg, 2010, 5: 99.

17. Porra L, Bayat $S$, Malaspinas I, et al. Pressure-regulated volume control vs. volume control ventilation in healthy and injured rabbit lung: An experimental study. Eur J Anaesthesiol. 2016 Oct;33(10):76775. 
18. Kido T, Morimoto $\mathrm{Y}$, Yatera $\mathrm{K}$, et al. The utility of electron microscopy in detecting asbestos fibers and particles in BALF in diffuse lung diseases. BMC Pulm Med. 2017 Apr 21;17(1):71.

19. Wang Z, Yang W, Yang P, et al. Effect of Radix Stemonae concentrated decoction on the lung tissue pathology and inflammatory mediators in COPD rats. BMC Complement Altern Med. 2016 Nov 10;16(1):457.

20. Sulaiman I, Cushen B, Greene G, et al. Objective Assessment of Adherence to Inhalers by Patients with Chronic Obstructive Pulmonary Disease. Am J Respir Crit Care Med. 2017 May 15;195(10):13331343.

\section{Figures}

\section{A BALF}

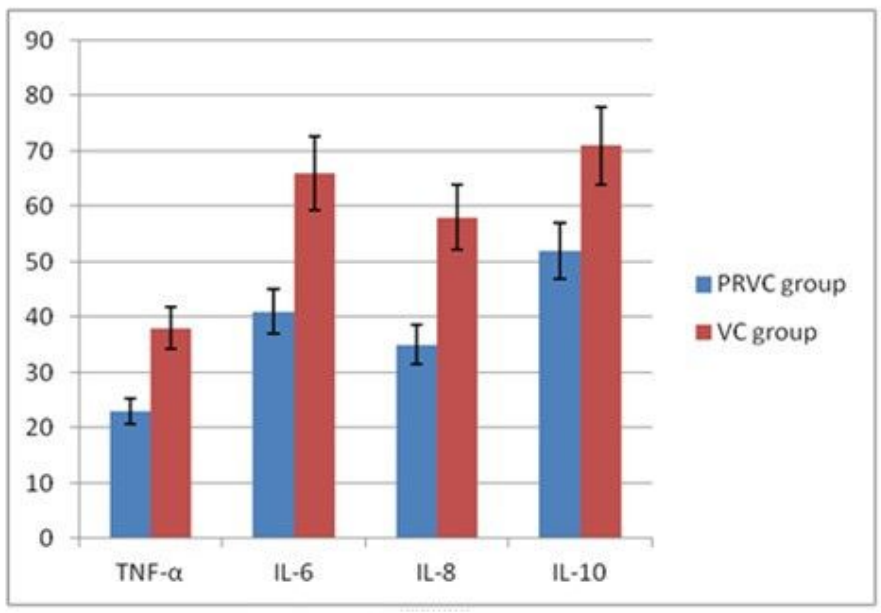

\section{B blood sample}

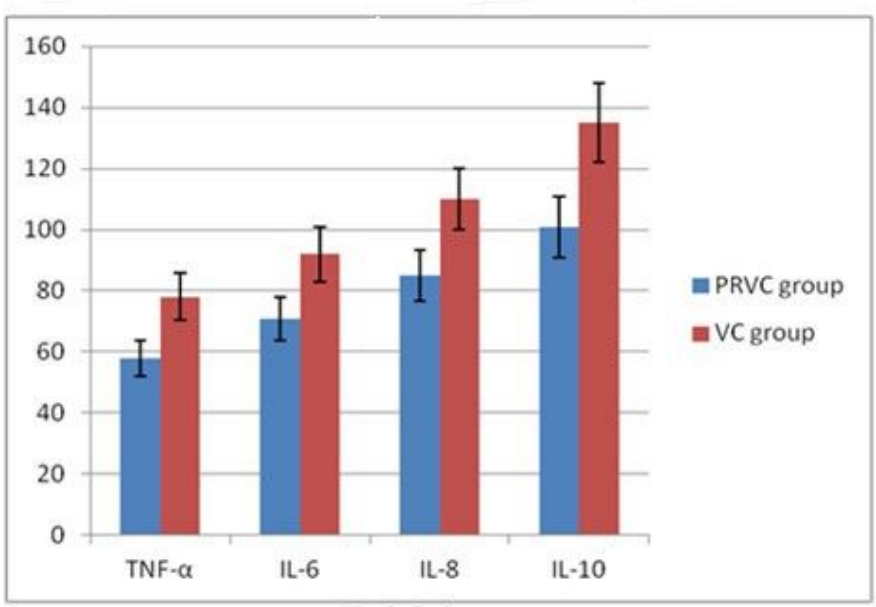

\section{Figure 1}

Two ventilation modes on pulmonary and systamic inflammatory responses: A, the levels of TNF-, IL-6, IL8 and IL-10 from BALF in the PRVC group were significantly lower than those in the VC group $(p<0.05)$. B, 
the levels of TNF-, IL-6, IL-8 and IL-10 from blood sample in the PRVC group were significantly lower than those in the VC group $(p<0.05)$. 